\title{
Violência, Funções Executivas e Rendimento Acadêmico em Estudantes Universitários
}

\author{
Leandro Jorge Duclos da Costa \\ Universidade Estadual de Goiás e Faculdade Estácio de Sá de Goiás, Goiânia-GO, Brasil \\ Daniela Sacramento Zanini \\ Pontifícia Universidade Católica de Goiás, Goiânia-GO, Brasil \\ Larissa de Oliveira e Ferreira \\ Faculdade Estácio de Sá de Goiás, Goiânia-GO, Brasil \\ Karina Junqueira Leão \\ Centro de Reabilitação e Readaptação Dr. Henrique Santillo - CRER/GO, Goiânia-GO, Brasil
}

\section{RESUMO}

O presente artigo teve como objetivo avaliar impactos da vitimização, no último ano e ao longo da vida, no desempenho acadêmico no ensino superior a partir da análise das funções executivas. Participaram do estudo 90 estudantes com idade de 19 a 24 anos. Os instrumentos utilizados foram o Five Digit Test (FDT) e o questionário Juvenile Victimization Questionnaire (JVQ). Foram avaliados a violência ocorrida no último ano e ao longo da vida, as funções executivas (FE) e o rendimento acadêmico (CR). Os resultados apontaram que indivíduos que sofreram violência por maus-tratos e sexual no último ano apresentaram CR mais baixo em relação ao grupo que não sofreu violência. Violência por maus-tratos e violência sexual também correlacionaram positivamente com funções executivas, tanto nos processos automáticos quanto nos controlados. Os dados apontam que sofrer violências têm repercussão nas funções executivas e desempenho acadêmico na vida adulta.

Palavras-chave: funções executivas; rendimento acadêmico; violência.

\section{ABSTRACT - Violence, Executive Functions and Academic Achievement in University Students}

This study aimed to evaluate the impact of victimization in the previous year and throughout life on academic performance in higher education from the analysis of executive functions. The study included 90 students aged 19 to 24 years. The instruments used were the Five Digit Test (FDT) and the Juvenile Victimization Questionnaire (JVQ). Violence suffered in the previous year and throughout the lifetime, executive functions (EFs) and academic achievement (AC) were assessed. The results showed that individuals that had suffered abuse and sexual violence in the previous year had lower AC compared to the group that had not suffered violence. Violence through ill-treatment and sexual violence also positively correlated with executive functions in both automatic and controlled processes. The data indicate that suffering violence has repercussions on the executive functions and academic performance in adulthood.

Keywords: executive functions; academic achievement; violence.

\section{RESUMEN - Violencia, Funciones Ejecutivas y Rendimiento Académico en Estudiantes Universitarios}

Este artículo tuvo como objetivo evaluar el impacto de la victimización en el rendimiento académico en la educación superior, en el último año y a lo largo de la vida, a partir del análisis de las funciones ejecutivas. El estudio se realizó con 90 estudiantes con edades entre 19 y 24 años. Los instrumentos utilizados fueron el Five Digit Test (FDT) y el cuestionario Juvenile Victimization Questionnaire (JVQ). Se evaluó la violencia vivida en el último año y a lo largo de la vida, las funciones ejecutivas (FE) y el rendimiento académico (CR). Los resultados mostraron que las personas que sufrieron abuso y violencia sexual en el último año tuvieron un CR más bajo en comparación con el grupo que no sufrió violencia. La violencia por maltrato y violencia sexual también se correlacionaron positivamente con las funciones ejecutivas tanto en procesos automáticos como en los controlados. Los datos indican que sufrir violencia genera repercusiones en las funciones ejecutivas y el rendimiento académico en la edad adulta.

Palabras clave: funciones ejecutivas; rendimiento académico; violencia.

A violência pode ser entendida como qualquer ato intencional humano baseado na coerção por uso de poder ou condição física para ameaçar, agredir pessoas, grupos ou a si próprio, com forte potencial lesivo, dano psicológico, privação de direitos e, em casos extremos, morte (OMS, 2014). O Brasil ocupa o décimo lugar na 
escala mundial de mortes causadas por violência, de acordo com estudos do Mapa da Violência divulgado no ano de 2016. Esse estudo mostra que os tipos de violência mais recorrentes no país são feminicídio, estupro, racismo, violência policial, violência contra homossexuais, abuso na infância e na adolescência, violência no trânsito, violência no campo, narcotráfico, roubos e maus-tratos (Waiselfisz, 2016).

A experiência de situações de violência que causam danos às pessoas por ações de outros seres humanos, que violaram as normas sociais com potencial especial para causar traumas é denominada vitimização. Já revitimização é o fenômeno de repetição do mesmo tipo de violência já sofrida anteriormente pelo indivíduo (Finkelhor, 2007). A exposição a contextos violentos pode afetar o comportamento humano em diferentes etapas da vida por comprometer diretamente as esferas sociais da vida diária, relações profissionais futuras e habilidades cognitivas no decorrer da vida (Brancalhone, Fogo, \& Williams, 2004; Nuñes Carvalho, 2016).

O comportamento humano pode ser compreendido como constituído de três esferas interligadas: cognição, emocionalidade e funcionamento executivo ou funções executivas (FE) (Lezak, 1995). A cognição, conhecida também por atividade cerebral, refere-se a uma interpretação de conteúdos internos adquiridos ao longo das nossas vivências, envolvendo as funções cognitivas superiores (percepção, atenção, memória, linguagem, comportamentos físicos e emocionais). A emocionalidade, por sua vez, tende a ser associada à motivação e ao sentimento pelo qual o indivíduo executa a atividade proposta, enquanto as funções executivas teriam como propósito identificar o principal objetivo ou intenção de um comportamento, organizar os passos necessários para a execução da tarefa e verificar se o desempenho está efetivo.

As funções executivas (FE) envolvem a seleção e integração de informações atuais com informações previamente memorizadas, como o planejamento, o monitoramento e a flexibilidade cognitiva (Gazzaniga, Ivry, \& Magnun, 2002; Lezak, 1995). Seu objetivo é permitir ao indivíduo construir e direcionar metas em geral, ponderar atitudes e comportamentos e planejar e replanejar percursos mediantes a situações-problema (Lezak, 2004). Há muitas possibilidades para caracterizar e definir as FE, mas o modelo proposto por Miyake et al. (2000) tem sido internacionalmente reconhecido como adequado. Nesse modelo, as FE teriam três componentes básicos: memória de trabalho, controle inibitório e flexibilidade cognitiva.

A memória de trabalho é responsável pela manutenção de informações em curto espaço de tempo, como guardar um número de telefone. O controle inibitório está intimamente direcionado para controlar impulsos mentais relacionados ao controle emocional, por exemplo, o controle das atitudes quando se está insatisfeito com algo. O terceiro componente, flexibilidade cognitiva, refere-se à capacidade de interferir e modificar percursos previamente estabelecidos com base em tomadas de decisão e resolução de problemas, por exemplo, alterar a regra de um jogo para que possa contemplar maior número de participantes (Miyake et al., 2000).

De acordo com o modelo proposto por Miyake et al. (2000), as FE estão relacionadas à capacidade de processar e armazenar informações simultaneamente, manejar conhecimentos, controlar comportamentos e realizar planejamentos. Pode-se constatar que os processos ligados as FE podem impactar e tem direta relação, não só com o desempenho acadêmico de estudantes, mas também com as vivências de estressores significativos.

O rendimento acadêmico é uma das formas de averiguar processos de aprendizagem, sendo comumente expresso por indicadores quantitativos (notas) durante determinado período de tempo, embora certamente não é o único meio para sua avaliação. $O$ estudo sobre a aprendizagem relaciona o processo de aprender às capacidades de planejamento e regulação da própria atividade em função de determinados objetivos, ou seja, às próprias FE (Corso, Sperb, Jou, \& Salles, 2013). Com base nessas associações, prejuízo em funções executivas também poderiam dificultar os processos de aprendizagem e o rendimento acadêmico, como ilustrado em alguns estudos empíricos realizados no contexto do Brasil, voltados a analisar o possível efeito da violência sobre esses processos internos.

Indivíduos expostos a contextos violentos podem sofrer comprometimento nas funções executivas e, de modo associado, em seu desempenho acadêmico. Brancalhone et al. (2004) examinaram 30 crianças, com idade entre sete e 11 anos, sendo 15 crianças do ensino fundamental expostas à violência conjugal e 15 crianças não expostas à violência, do mesmo sexo e idade, escolhidas nas mesmas salas de aula. Os professores responderam à Escala de Avaliação da Performance Acadêmica (EAPA) e forneceram o Boletim Escolar dessas crianças. O Teste do Desempenho Escolar (TDE) foi aplicado para constatar as áreas acadêmicas em que essas crianças apresentavam eventuais dificuldades. Das 15 crianças expostas à violência, 14 presenciaram pelo menos um episódio de agressão da mãe, e sete estavam convivendo com violência conjugal há mais de cinco anos. Os resultados da EAPA foram significativamente menores para o grupo exposto à violência, com a média de 52,9 pontos contra 67,8 do grupo de crianças não expostas. No entanto, nas análises dos resultados dos conceitos do Boletim Escolar e do TDE, as diferenças entre os grupos não foram estatisticamente significativas.

A partir da investigação dos microdados do Sistema de Avaliação da Educação Básica (SAEB), Oliveira e Ferreira (2013) avaliaram possíveis efeitos da violência em escolas sobre o desempenho acadêmico de alunos. Focalizaram estudantes do nono ano do ensino fundamental, tendo como base as informações do ano de 2011. 
Seus resultados indicaram claramente que a violência escolar reduziu a probabilidade de adequado desempenho em atividades acadêmicas pelos discentes.

Ainda investigando crianças do ensino fundamental (entre 10 e 11 anos), Medeiros, Torro-Alves, Malloy-Diniz e Minervino (2016) avaliaram possível impacto do bullying no funcionamento efetivo infantil. Participaram da pesquisa 60 crianças que foram divididas em quatro grupos; agressores, vítimas, vítimas que se tornaram agressivas e grupo controle. $\mathrm{O}$ grupo de crianças vítimas e agressoras mostraram dificuldade nos processos de tomada de decisões. O estudo apontou que os agressores evidenciaram comprometimento no funcionamento executivo geral, ao passo que as vítimas sinalizaram, comparativamente, menor rendimento cognitivo (funções executivas relacionadas a cognição). Assim, concluíram que, além das variáveis sociais e culturais, os fatores neurocognitivos e emocionais tendem a influenciar o comportamento das crianças em situações de bullying.

Por sua vez, Nuñes Carvalho (2016) buscou avaliar o perfil cognitivo global, bem como a prevalência de prejuízo intelectual em amostra de crianças de 6 a 12 anos, vítimas de maus-tratos. Encontrou diferenças estatisticamente significativas entre os grupos de crianças em todas as funções avaliadas, com a presença de importante prejuízo intelectual no grupo com histórico de maus-tratos.

$\mathrm{Na}$ fase adulta do desenvolvimento, esses estudos sobre rendimento acadêmico e funcionamento executivo são mais escassos no Brasil, de acordo com pesquisa realizada em fevereiro de 2019 no Portal CAPES, com os seguintes descritores: "victimization and executive function and young adults". Cabe, no entanto, apontar a pesquisa realizada por Yang et al. (2013) . O estudo buscou investigar o comprometimento das funções executivas, a partir de medidas neuropsicológicas (testes neuropsicológicos) e comportamentais (questionários e inventários), comparando estudantes polivitimizados com estresse pós-traumático e polivitimizados sem estresse pós-traumático.

Os estudantes que participaram da pesquisa eram chineses entre 18 e 21 anos $(n=259)$. Os voluntários foram divididos em quatro grupos conforme seu histórico de vida: revitimizados com estresse pós-traumático, revitimizados sem estresse pós-traumático, não revitimizados e não vitimizados. Os instrumentos utilizados para avaliação das funções executivas foram: Behavior Rating Inventory of Executive Funcionts versão para adultos (BRIEF A), que se trata de um questionário usado para avaliar FE em adolescentes e adultos a partir de 18 anos; Cambridge Neuropsychological Testing Automated Battery (CANTAB), que se trata de um teste neuropsicológico informatizado que é dividido em dois subtestes de treino (triagem) e quatro subtestes que avaliam atenção sustentada, flexibilidade cognitiva, memória operacional, controle inibitório e planejamento. Para identificação da revitimização e polivitimização, foi usado o Juvenile Victimization Questionaire (JVQ) e para identificação do estresse pós-traumático foi usado o PCL-C, um inventário de autorrelato que mapeia os sintomas do estresse pós-traumático a partir dos critérios do DSM IV.

Os resultados apontaram que os indivíduos revitimizados com e sem estresse pós-traumático tiveram pior desempenho na escala BRIEF-A que avalia aspectos comportamentais das FE. O grupo revitimizados com estresse pós-traumático, quando comparado com o grupo não revitimizados, alcançou resultado inferior nos testes neuropsicológicos (CANTAB) e nas escalas comportamentais (BRIEF-A), nos componentes de controle inibitório, flexibilidade cognitiva, controle emocional e memória de trabalho. Seus resultados apontaram correlação negativa e estatisticamente significativa entre jovens adultos revitimizados e flexibilidade cognitiva. Dentro desse trabalho, as conclusões apontaram que, quanto mais experiência de revitimizações com crimes sexuais, pior o desempenho na flexibilidade cognitiva aferida no FDT (pontuações inferiores no quesito alternância).

Em conjunto, as investigações científicas descritas apontam que a violência tende a impactar, de forma significativa e negativa, o funcionamento emocional e cognitivo da criança e do adolescente, podendo ter desdobramentos na vida adulta. Nesse sentido, o presente estudo busca avaliar eventuais impactos da vitimização na infância e adolescência e sua possível relação com o desempenho acadêmico no ensino superior e as funções executivas em jovens adultos. De forma específica, procurou-se verificar a relação entre vitimização e desempenho acadêmico e possível associação entre vitimização e funcionamento executivo.

\section{Método}

\section{Participantes}

A amostra consiste em 90 alunos, com idades entre 19 e 24 anos, de ambos os sexos, voluntários regularmente matriculados em uma Instituição de Ensino Superior da cidade de Goiânia (GO). Essa instituição foi selecionada segundo critério de acessibilidade aos pesquisadores, constituindo amostra de conveniência.

Os critérios de inclusão no estudo foram: faixa etária entre 19 e 24 anos, regulamente matriculado entre o segundo e quinto período do curso de graduação; não possuir diagnóstico autodeclarado de transtorno do desenvolvimento ou transtornos neuropsiquiátricos; participação voluntária na pesquisa, tendo assinado o Termo de Consentimento Livre e Esclarecido. Os critérios de exclusão foram: possuir alterações comportamentais, de linguagem, visuais e motoras que inviabilizasse a execução dos instrumentos propostos e incompletude das respostas na coleta de dados.

\section{Instrumentos}

Juvenile Victimization Questionnaire (JVQ). Questionário específico para mapear tipos de violência, 
vitimização e revitimização sofridos ao longo da vida e/ ou no último ano. Esse instrumento de investigação foi estruturado pelos pesquisadores Finkelhor, Hamby, Ormord e Turner (2005), nos Estados Unidos da América, e traduzido e adaptado, para o contexto brasileiro, pelas pesquisadoras Faria e Zanini (2011), sem prejuízos da estrutura original. O instrumento pode ser utilizado com crianças, adolescentes e adultos. Versa sobre 34 situações de violência que podem ser agrupadas em cinco tipos de crivos: crimes convencionais, maus-tratos, violência entre pares, vitimização sexual e vitimização testemunhada ou indireta.

Os crimes convencionais são aqueles comumente identificados pelas autoridades policiais. Esse crivo examina ocorrências de agressões, roubos, vandalismo, tentativa de agressão e preconceito. Os maus-tratos são caracterizados pela vivência de situações de violência ligadas diretamente a família ou a funcionários ligados ao cuidar domiciliar. Nesse crivo, avalia-se também a negligência, sequestro por familiar, abusos físicos e psicológicos. O terceiro crivo trata das vitimizações por pares, que são as agressões que ocorrem por indivíduos da mesma faixa etária, normalmente entre estudantes. Nesse crivo, são examinadas agressões por prática de bullying, ataques por grupos e violência física e psicológica entre pares. $\mathrm{O}$ quarto crivo trata da vitimização sexual, que são agressões relacionadas a questões ligadas à sexualidade e engloba agressões sexuais por adulto, por pares, assédio sexual, estupro ou tentativa de estupro e assédio verbal ligado à sexualidade. As vitimizações indiretas se referem a testemunhar violência sofrida por outras pessoas. Nesse crivo, são examinadas questões, como testemunhar violência doméstica, presenciar os pais agredirem os irmãos ou cônjuge, testemunhar ataques, roubos, assassinato, tiroteios, terrorismo e exposição a conflitos étnicos (Hamby, Finkelhor, Ormrod, \& Turner, 2004).

O participante responde ao JVQ por meio de um checklist em que informa se vivenciou ou não (marcando com um " $\mathrm{x}$ " no sim ou no não) cada uma das situações de violência descritas em duas colunas. A primeira coluna trata das vivências ocorridas no último ano e a outra coluna, para experiências de violência ao longo da vida. A somatória dos itens correspondentes a cada crivo gera a pontuação total do JVQ.

Five Digit Test (FDT). Teste psicológico destinado a avaliar as funções executivas. Foi desenvolvido pelo pesquisador Manuel Sedó (2007) e reconhecido pela Sociedade Neurospicológica de Massachutts (EUA) por sua validade em aferir leitura, contagem, alternância e flexibilidade cognitiva que são aspectos relevantes a serem observados na avaliação das FE. Nesse estudo, foi utilizada a versão brasileira adaptada por Sedó, Paula e Malloy-Diniz (2015), que apresenta dados psicométricos satisfatórios e estudos normativos para as cinco regiões brasileiras, na faixa etária de 5 a 95 anos (Sedó, Paula \& Malloy-Diniz 2015).
O teste se apresenta em quatro etapas, na forma de figuras e números. Na primeira fase, o examinador apresenta ao sujeito 50 quadrados contendo números e solicita ao examinando que leia o número que está dentro do quadrado. Nessa etapa, há congruência entre o número grafado e a quantidade de números representados no quadrado. $\mathrm{Na}$ segunda fase, o sujeito se depara com 50 quadrados contendo pequenas figuras (como um asterisco). A tarefa consiste em contar quantas figuras há em cada quadrado. Na terceira fase, a tarefa consiste em contar quantos números há em cada um dos 50 quadrados. No entanto, nessa etapa, há incongruência entre o número representado no quadrado e a sua quantidade. $\mathrm{Na}$ última etapa, o sujeito se depara com uma situação semelhante a anterior, na qual deve contar quantos números há em cada quadrado, havendo incongruência entre o número representado no quadrado e a sua quantidade. No entanto, em alguns quadrados há uma moldura escura. Quando o sujeito encontra essa moldura deve falar qual o número representado e não quantos números há no quadrado. Assim, nessa última etapa, além do efeito Stroop, há também a medida de flexibilidade cognitiva, visto que o sujeito deverá alternar entre as duas estratégias cognitivas durante a tarefa.

Sedó, Paula e Malloy Diniz (2015) afirmam que as partes de leitura e contagem medem diversas variáveis em um mesmo processo, principalmente atenção, posteriormente, a fluidez e a ação continuada do sujeito. As partes de escolha e alternância medem o mesmo processo mental, porém, acrescentam algumas mudanças no processo de tomada de decisão da resposta. Nas escolhas, intervém o controle inibitório e, na alternância, aparece a inibição de uma rotina e a ativação de outra. Todos esses processos são considerados componentes relevantes na avaliação das FE.

Desempenho acadêmico discente. A avaliação do desempenho acadêmico foi feita pela variável Coeficiente de rendimento (CR). A instituição avaliada considera rendimento acadêmico satisfatório para aprovação em uma disciplina nota igual ou superior a 6,0, em uma escala de 0 a 10 . Ao longo do curso, a somatória das médias finais obtidas em cada disciplina dividido pelo número de disciplinas cursadas gerou o $\mathrm{CR}$ do aluno. Dessa forma, quanto maior o CR, melhor o desempenho acadêmico desse aluno. As pontuações dos participantes foram agrupadas em três níveis ( 1 aqueles com baixo índice de CR, 2 aqueles com médio e 3 aqueles com alto nível de CR), constituindo três subgrupos de estudantes para análise nas demais variáveis de interesse no estudo.

\section{Procedimentos}

A pesquisa foi avaliada e aprovada por um Comitê de Ética em Pesquisa (CEP), com protocolo CAAE 70171617.5.0000.0037 e número do parecer 2.223.772. Após essa etapa, o gerente acadêmico da instituição de 
ensino convidada ao estudo ofereceu sua autorização para a coleta de dados.

Os contatos com os participantes da pesquisa ocorreram nos dias e horários das aulas, nas turmas do segundo, terceiro, quarto e quinto períodos do curso de Educação Física da instituição colaboradora. A pesquisa foi apresentada em todas as turmas elegíveis e, após esse procedimento, foram selecionados os alunos com base nos critérios de inclusão, convidando-os ao estudo. Os que livremente concordaram com sua participação, assinaram o TCLE, ficando com uma via desse documento.

A coleta de dados ocorreu na própria sala de aula, em ajustes com os responsáveis pelas turmas, nos períodos matutino e noturno. Os instrumentos foram aplicados coletivamente, em um único encontro, seguindo diretrizes técnicas específicas de seus manuais.

\section{Análise dos Dados}

Cada protocolo individual foi devidamente codificado pelos pesquisadores, conforme suas normas técnicas. Esses dados foram lançados no Statistical Package for the Social Sciences (SPSS), versão 24.0, para as análises estatísticas. Inicialmente verificou-se o tipo de distribuição dos resultados, checando-se sua normalidade. $\mathrm{O}$ teste estatístico de hipótese para verificação de normalidade univariada denominado Shapiro Wilk foi aplicado individualmente ao conjunto de variáveis de coeficiente de rendimento (CR), FDT e violências (crimes convencionais, maus-tratos, violência entre pares, vitimização sexual e vitimização testemunhada e indireta), confirmando-se distribuição normal dos dados. Os resultados foram inicialmente sistematizados de forma descritiva e, posteriormente, realizaram-se análises do FDT, CR em sua relação com as variáveis de violência retiradas do JVQ. Recorreu-se à estratégia de comparação de médias (ANOVA) e subsequentemente teste de Bonferroni para comparação múltipla de médias, seguidas pelas análises de correlação de Pearson $(r)$. Foi considerado o intervalo de confiança de $95 \%$ em todas as análises realizadas.

\section{Resultados}

Os resultados serão apresentados em duas partes. $\mathrm{Na}$ primeira, constam as variáveis sobre violência e desempenho acadêmico (CR), seguida pela análise entre violência e funcionamento executivo $(\mathrm{FE})$.

\section{Violência e Desempenho Acadêmico (CR)}

Para verificar a existência de diferenças entre grupos com baixo, médio ou alto coeficiente de rendimento, foi realizada a categorização da variável CR por meio do quartil. Dessa forma, as pontuações dos participantes foram agrupadas em três níveis (1 aqueles com baixo índice de CR, 2 aqueles com médio e 3 aqueles com alto nível de CR). Posteriormente, esses três grupos foram comparados em seus indicadores médios nas variáveis relativas à vitimização (JVQ). A Tabela 1 apresenta os dados de comparação de média (ANOVA) entre os crivos de vitimização e os diferentes grupos de CR.

Tabela 1

Indicadores de Vitimização (Média e Desvio Padrão) nos Estudantes ( $n=90)$ em Função do Rendimento Acadêmico

\begin{tabular}{lccccc}
\hline \multirow{2}{*}{ Vitimização* } & \multicolumn{3}{c}{ Rendimento acadêmico (CR) } & \multicolumn{2}{c}{ Comparação estatística } \\
\cline { 2 - 6 } & $\begin{array}{c}\text { Baixo CR } \\
(\mathrm{n}=36)\end{array}$ & $\begin{array}{c}\text { Médio CR } \\
(\mathrm{n}=31)\end{array}$ & $\begin{array}{c}\text { Alto CR } \\
(\mathrm{n}=23)\end{array}$ & $\mathrm{F}$ & $p$ \\
\hline No último ano de vida & & & & \\
\hline Convencional & $7,05(1,05)$ & $6,98(0,84)$ & $7,04(0,66)$ & 0,41 & 0,96 \\
Maus-tratos & $7,20(0,13)^{\mathrm{a}}$ & $7,10(0,14)$ & $6,66(0,97)^{\mathrm{b}}$ & 3,19 & 0,05 \\
Pares & $7,14(0,68)$ & $6,84(0,92)$ & $6,92(1,14)$ & 1,12 & 0,33 \\
Sexual & $7,29(0,61)^{\mathrm{a}}$ & $7,12(0,77)$ & $6,67(1,00)^{\mathrm{b}}$ & 3,62 & 0,03 \\
Indireta & $7,04(0,81)$ & $7,48(0,68)$ & $6,92(0,85)$ & 2,60 & 0,08 \\
Ao longo da vida & & & & & \\
\hline Convencional & $6,94(0,85)$ & $6,95(0,71)$ & $7,05(0,85)$ & 0,82 & 0,92 \\
Maus-tratos & $7,31(0,70)$ & $7,13(0,92)$ & $6,96(0,84)$ & 0,80 & 0,44 \\
Pares & $7,17(0,60)$ & $7,16(0,57)$ & $6,94(0,95)$ & 0,71 & 0,49 \\
Sexual & $7,22(0,66)$ & $7,02(0,90)$ & $6,96(0,85)$ & 0,51 & 0,60 \\
Indireta & $6,82(1,13)$ & $7,39(0,27)$ & $7,03(0,83)$ & 0,41 & 0,65 \\
\hline
\end{tabular}

Nota. *Dados médios e desvio padrão (entre parênteses).; (a) e (b)=subgrupos com diferença estatisticamente significativa

A comparação entre subgrupos de rendimento acadêmico revelou diferença estatisticamente significativa rem relação à violência por maus-tratos no último ano de vida $(p=0,05)$. Os estudantes com maior desempenho 
escolar foram aqueles com menor relato de experiências de maus-tratos (b). A violência do tipo sexual no último ano também diferenciou os estudantes em termos de rendimento acadêmico $(p=0,03)$. Os adultos jovens com menor vitimização sexual (b) foram aqueles que apresentaram maior média no CR. Para avaliar a relação entre as variáveis vitimizações e desempenho acadêmico (CR), fez-se análise de correlação de Pearson. Esses dados compõem a Tabela 2.
Embora os valores de correlação identificados sejam moderados, houve relevante associação entre as experiências de vitimização no último de ano e o rendimento acadêmico na graduação. Foram encontradas correlações negativas e significativas entre as variáveis violência por maus-tratos e sexual no último ano e CR. Os achados indicaram que quanto mais vitimizações do tipo maus-tratos e sexual o adulto jovem vivenciou menor seu rendimento acadêmico.

Tabela 2

Indicadores de Correlação entre Vitimizações e Coeficiente de Rendimento dos Estudantes (n=90)

\begin{tabular}{lcc}
\hline \multicolumn{1}{c}{ Vitimização } & No último ano & Ao longo da vida \\
\hline Convencional & $-0,01$ & 0,04 \\
Maus-tratos & $-0,24^{*}$ & $-0,14$ \\
Pares & $-0,13$ & $-0,12$ \\
Sexual & $-0,27^{*}$ & $-0,10$ \\
Indireta & $-0,11$ & 0,03 \\
\hline
\end{tabular}

Nota. ${ }^{*}$ Correlação significativa $p \leq 0,05$

\section{Violência e Funções Executivas (FE)}

Para análise da possível associação entre indicadores de violência (JVQ) e o funcionamento executivo, conforme dados do FDT, realizou-se análise de correlação entre esses achados. Os dados estão apresentados na Tabela 3.

É importante ressaltar algumas explanações sobre as correlações positivas identificadas entre as variáveis do FDT e as vitimizações. Nas quatro etapas do FDT (leitura, contagem, escolha e alternância), o tempo é a medida principal. Desse modo, para a interpretação da pontuação bruta, entende-se que quanto maior o tempo gasto para realizar o teste pior é seu desempenho. Em outras palavras, quanto mais tempo gasto na realização de cada etapa, pior é o desempenho do sujeito e maior seu comprometimento. Nesse mesmo sentido, é feita a interpretação da quantidade de erros e o desvio padrão. Quanto maior a quantidade de erros e quanto maior o desvio padrão, pior o desempenho do sujeito e maior seu comprometimento. Portanto, as correlações tornaram-se positivas entre as variáveis em foco, apontando que quanto mais vitimização, maiores foram as pontuações no FDT (bruta, erro e desvio padrão), evidenciando maiores comprometimentos.

Tabela 3

Índices de Correlação entre as Vitimizações e as Funções Executivas em Jovens Adultos $(n=90)$

\begin{tabular}{|c|c|c|c|c|c|c|c|c|c|c|}
\hline \multirow{3}{*}{$\begin{array}{l}\text { Indicador FDT } \\
\text { (FE) }\end{array}$} & \multicolumn{10}{|c|}{ Vitimização } \\
\hline & \multicolumn{5}{|c|}{ No último ano } & \multicolumn{5}{|c|}{ Ao longo da vida } \\
\hline & Conv. & Maus-Tratos & Pares & Sexual & Indireta & Conv. & $\begin{array}{l}\text { Maus- } \\
\text { Tratos }\end{array}$ & Pares & Sexual & Indireta \\
\hline A.B & 0,09 & 0,10 & 0,11 & $0,21^{*}$ & 0,18 & $-0,1$ & $-0,04$ & $-0,03$ & $-0,03$ & 0,04 \\
\hline A.DP & 0,01 & 0,09 & 0,09 & 0,17 & $0,22^{*}$ & $-0,13$ & 0,02 & 0,01 & 0,04 & 0,13 \\
\hline A.E.B & $-0,03$ & 0,1 & 0,13 & 0,04 & $0,24^{*}$ & $-0,19$ & $-0,15$ & $-0,05$ & $-0,05$ & $-0,07$ \\
\hline A.E.DP & $-0,02$ & 0,19 & 0,19 & $0,28^{* *}$ & 0,05 & $-0,24^{*}$ & $-0,16$ & $-0,05$ & $-0,04$ & $-0,11$ \\
\hline I.B & $-0,11$ & $-0,09$ & 0 & 0 & $-0,08$ & 0,05 & $-0,07$ & 0,03 & $-0,08$ & 0,03 \\
\hline I.DP & $-0,11$ & $-0,13$ & 0,04 & 0,03 & $-0,03$ & 0,06 & $-0,07$ & 0 & $-0,07$ & 0,03 \\
\hline F.B & $-0,04$ & 0,11 & $-0,01$ & 0,13 & 0,07 & $-0,05$ & $-0,04$ & 0 & $-0,05$ & 0,13 \\
\hline F.DP & $-0,03$ & $-0,06$ & 0,01 & 0,12 & 0,02 & $-0,02$ & $-0,15$ & 0,01 & $-0,04$ & 0,13 \\
\hline L.B & 0,17 & $-0,03$ & 0,16 & 0,12 & 0,15 & $-0,08$ & $-0,02$ & $-0,03$ & 0,02 & $-0,13$ \\
\hline L.DP & $0,26^{*}$ & 0,02 & 0,10 & 0,18 & 0,11 & $-0,01$ & 0,03 & $-0,08$ & $-0,09$ & 0,01 \\
\hline L.E.B & 0,03 & $0,28^{* *}$ & 0,03 & $0,35^{* *}$ & 0,01 & $-0,01$ & 0,19 & 0,03 & $0,37^{* *}$ & $-0,02$ \\
\hline L.E.DP & 0,13 & $0,21^{*}$ & 0 & $0,32^{* *}$ & 0 & 0 & 0,19 & 0,05 & $0,32^{* *}$ & 0,02 \\
\hline C.B. & 0,10 & $-0,02$ & $0,24^{*}$ & 0,20 & 0,10 & $-0,13$ & $-0,03$ & $-0,09$ & $-0,02$ & $-0,02$ \\
\hline
\end{tabular}


Tabela 3 (continuação)

Índices de Correlação entre as Vitimizações e as Funções Executivas em Jovens Adultos (n=90)

\begin{tabular}{|c|c|c|c|c|c|c|c|c|c|c|}
\hline \multirow{3}{*}{$\begin{array}{c}\text { Indicador FDT } \\
(\mathrm{FE})\end{array}$} & \multicolumn{10}{|c|}{ Vitimização } \\
\hline & \multicolumn{5}{|c|}{ No último ano } & \multicolumn{5}{|c|}{ Ao longo da vida } \\
\hline & Conv. & Maus-Tratos & Pares & Sexual & Indireta & Conv. & $\begin{array}{l}\text { Maus- } \\
\text { Tratos }\end{array}$ & Pares & Sexual & Indireta \\
\hline C.DP & 0,07 & 0 & $0,24^{*}$ & 0,11 & 0,08 & $-0,09$ & 0 & $-0,05$ & $-0,07$ & $-0,02$ \\
\hline C.E.B & $-0,03$ & 0,02 & 0 & $0,34^{* *}$ & $-0,07$ & 0,04 & $-0,03$ & $0,26^{*}$ & 0 & $-0,19$ \\
\hline C.E.DP & $-0,02$ & $-0,02$ & $-0,01$ & $0,28^{*}$ & $-0,08$ & 0,03 & $-0,06$ & $-0,01$ & $0,22^{*}$ & $-0,19$ \\
\hline E.B & $-0,06$ & $-0,11$ & 0,16 & 0,12 & 0,08 & $-0,03$ & $-0,08$ & 0 & $-0,06$ & $-0,10$ \\
\hline E.DP & 0,09 & $-0,14$ & 0,15 & 0,07 & 0,06 & 0,03 & $-0,02$ & 0,05 & $-0,05$ & 0,01 \\
\hline E.E.B & 0,01 & 0,05 & 0,12 & $-0,01$ & 0,10 & $-0,10$ & $-0,12$ & 0,01 & 0,01 & $-0,06$ \\
\hline E.E.DP & 0,06 & 0,15 & 0,14 & $-0,01$ & 0,10 & $-0,04$ & $-0,04$ & 0,06 & 0,06 & 0,01 \\
\hline
\end{tabular}

Nota. ${ }^{*}$ Correlação significativa $p \leq 0,05 ;{ }^{* *}$ Correlação significativa $p \leq 0,01 ;{ }^{* *}$ Sobre os dados apresentados acima é importante ressaltar que os processos de leitura e contagem avaliam processos controlados e atenção. Já os dados "Escolha" e "Inibição" avaliam controle inibitório. Por fim, "Alternância" e "Flexibilidade" avaliam flexibilidade cognitiva

Legenda. FE=Funções Executivas; Conv.=Convencional; Funções Executivas=LB (Leitura Bruto); L. DP (Leitura Desvio Padrão); L.E.B (Leitura Erro Bruto); L.E.DP (Leitura Erro Desvio Padrão); CB (Contagem Bruto); C. DP (Contagem Desvio Padrão); C.E.B (Contagem Erro Bruto); C.E.DP (Contagem Erro Desvio Padrão); EB (Escolha Bruto); E.DP (Escolha Desvio Padrão); E.E.B (Escolha Erro Bruto); E.E.DP (Escolha Erro Desvio Padrão); A.B (Alternância Bruto); A. DP (Alternância Desvio Padrão); A.E.B (Alternância Erro Bruto); A.E.DP (Alternância Erro Desvio Padrão); I.B (inibição Bruto); I. DP (Inibição Desvio Padrão); F.B (Flexibilidade Bruto); F. DP (Flexibilidade Desvio Padrão)

Os achados apontaram que houve correlação positiva entre todos os tipos de vitimizações ocorridas no último ano com o funcionamento executivo dos jovens. Essas correlações assumiram valores estatisticamente significativos (embora moderados) em violência convencional e maus-tratos, associando-se ao pior desempenho em leitura no FDT. Pode-se ressaltar que os jovens que sofreram violência convencional e maus-tratos apresentaram pior desempenho nos processos automáticos avaliados a partir da etapa de leitura do FDT. A leitura no FDT está diretamente relacionada com a capacidade de focar a atenção, considerada uma atividade básica para desenvolvimento dos demais componentes de funções executivas (memória de trabalho, controle inibitório e flexibilidade cognitiva). Portanto, os jovens que sofreram as vitimizações apresentadas acima têm um pior desempenho nos processos automáticos (atenção).

A violência entre pares correlacionou de forma positiva (moderada) com contagem no FDT. A contagem também é uma etapa que avalia processos automáticos relacionados com a atenção. Os dados apontaram que os universitários que vivenciaram violência entre pares apresentaram pior desempenho em contagem e, consequentemente, na capacidade em manter a atenção.

A violência indireta apresentou correlação positiva moderada com alternância no FDT. A etapa alternância faz parte da etapa de processos controlados, sendo a etapa de maior complexidade. Os processos controlados, especificamente a alternância, estão associados a melhor capacidade em atenção sustentada, assim como controle inibitório e flexibilidade cognitiva. Portanto, os jovens vitimizados por violência indireta apresentaram sinais de pior desempenho em dois dos três componentes básicos das FE, o que sugere comprometimento em FE.

Em complemento a esses dados, notou-se que indivíduos acometidos por crime sexual no último ano e ao longo da vida apresentaram correlação positiva em leitura, contagem e inibição no FDT. O abuso sexual no último ano apresentou correlações moderadas em leitura e contagem (processos automáticos), porém foram encontradas correlação significativas positivas entre processos controlados (inibição) avaliados no FDT. Assim, a violência sexual vivenciada no último ano comprometeu, moderadamente, os processos automáticos (atenção), enquanto, ao longo da vida, sinalizou prejudicar de modo significativo o processo de inibição (controle inibitório), conforme avaliação realizada a partir do FDT.

Os dados identificados foram sugestivos de que o abuso sexual pode impactar no desempenho acadêmico, assim como nas funções executivas. Mais especificamente, as funções mais atingidas foram a atenção e o controle inibitório nos jovens universitários.

\section{Discussão}

O presente estudo buscou avaliar impactos da vitimização e sua possível relação com o desempenho acadêmico no ensino superior e o funcionamento executivo em jovens universitários. Os resultados apontaram que a vivência de violência sexual se associou de modo positivo às $\mathrm{FE} \mathrm{e}$, de forma negativa, ao desempenho acadêmico desses estudantes. Messan-Moore, Walsh, e DiLillo (2010) encontraram resultados que vão nessa mesma direção. Eles avaliaram 752 mulheres universitárias que foram abusadas sexual ou fisicamente na infância. Os 
resultados apontaram o abuso sexual na infância como um fator de risco, ligado à revitimização na adolescência e na vida adulta. Apesar de não fazerem correlação com o desempenho acadêmico, o estudo indicou que a vitimização física ou sexual na infância pode trazer prejuízos na vida adulta.

A literatura científica já havia apontado, em estudos realizados no Brasil (Brancalhone et al., 2004; Oliveira \& Ferreira, 2013) que houve pior desempenho acadêmico em crianças vítimas de violência escolar e também em crianças e adolescentes que presenciaram violência conjugal. Essas pesquisas não avaliaram os impactos da vitimização na vida adulta, porém apontaram que a vitimização na infância e adolescência trazia prejuízos na vida acadêmica.

Quanto a relação da vivência de violência e o impacto nas FE, Medeiros, Torro-Alves, Malloy-Diniz e Minervino (2016) apontaram resultados que indicam que crianças vítimas de bullyng apresentaram comprometimento das funções executivas (componentes cognitivos), relacionados aos processos de desempenho acadêmico. Por sua vez, o estudo de De Bellis, Hooper, Spratt, e Woolley (2009) investigou possível associação entre abuso sexual e negligência nas funções executivas em crianças. Os autores avaliaram domínios neurocognitivos das habilidades motoras finas, linguagem visoespacial, memória, aprendizagem, atenção e funções executivas em crianças negligenciadas clinicamente saudáveis e sexualmente abusadas. Os resultados demonstraram que o grupo de crianças negligenciadas (com e sem estresse pós-traumático) obteve resultados neurocognitivos e desempenho acadêmico significativamente mais baixos do que o grupo controle.

A maioria dos estudos identificados no contexto brasileiro, nessa área de pesquisa, avaliaram o impacto da vitimização e revitimização (maus-tratos, violência entre pares, abuso físico e sexual) em crianças e adolescentes sobre o desempenho acadêmico e nas funções executivas. Porém, não avaliaram as consequências desses processos de vitimização em jovens adultos.
O estudo encontrado que avalia funções executivas e a vivência de violência em jovens adultos foi o de Yang et al. (2013) que apresentaram resultados similares ao da presente pesquisa. Seus achados apontaram que quanto mais revitimizações com crimes sexuais pior o desempenho na flexibilidade cognitiva. Este estudo ampara os resultados presentemente encontrados que apontaram que, quanto mais vivência de crime sexual ao longo da vida, pior o desempenho em FE, tanto nos processos automáticos quanto nos controlados, como alternância e flexibilidade cognitiva.

No presente estudo foi possível identificar o baixo desempenho no rendimento acadêmico em jovens adultos vitimizados e revitimizados em que a violência sofrida por maus-tratos e sexual ocorridas no último ano e também ao longo da vida, correlacionaram-se negativamente, somados ao comprometimento dos processos atencionais controlados, em termos de escolha e alternância, para o teste FDT. Os impactos da violência para o desempenho das funções executivas revelaram também que jovens adultos acadêmicos do ensino superior tiveram comprometimentos em diversas variáveis do FDT. Destaque deve ser dado para a influência negativa em indivíduos que sofreram violência sexual nos aspectos de leitura, alternância e contagem do FDT.

Os resultados evidenciaram que a violência ocorrida no último ano de vida tende a apresentar maior impacto nos aspectos cognitivos baseados nas FE. Mostraram ainda que a repetida experiência de violência tem um efeito ainda mais acentuado quando se trata de desempenho acadêmico. Assim, foi possível apresentar evidências empíricas de que o processo de aprendizagem avaliado segundo os elementos de FDT e CR são afetados pela vivência de violência, principalmente violência sexual. Logo, entende-se ser necessária continuidade às pesquisas, buscando conhecimento científico sobre a questão, atenção psicossocial aos indivíduos nas universidades e, em casos extremos, medidas protetivas para esses jovens para assegurar seu desempenho acadêmico.

\section{Referências}

Brancalhone, P. G., Fogo, J. C., \& Williams, L. C. A. (2004). Crianças expostas à violência conjugal: Avaliação do desempenho acadêmico. Psicologia Teoria e Pesquisa. 20(2) 113-117. doi: 10.1590/S0102-37722004000200003

Corso, H. V., Sperb, T., M., Jou, G. I., \& Salles, J. F., (2013). Metacognição e funções executivas: Relações entre os Conceitos e Implicações para a Aprendizagem. Psicologia: Teoria e Pesquisa, 29(1), 21-29. doi: 10.1590/S0102-37722013000100004

De Bellis, M.D., Hooper, S.R, Spratt, E.G., Woolley, D.P. (2009). Neuropsychological findings in childhood neglect and their relationships to pediatric PTSD. Journal of the International Neuropsychological Society, 15(6), 868-878. doi: 10.1017/S1355617709990464

Faria, M. R. G. V., Zanini, D. S. (2011). Análise da compreensão dos itens do Questionário de Vitimização (JVQ) após tradução para o português. Trabalho apresentado como Pôster na 63 Reunião Anual da SBPC, 10 a 15 de julho de 2011-UFG- Goiânia, GO. Retirado de http:// www.sbpcnet.org.br/livro/63ra/resumos/resumos/6155.htm.

Finkelhor, D., Ormrod, R. K. \& Turner, H. A. (2007). Re-victimization patterns in a national longitudinal sample of children and youth. Child Abuse \& Neglect, 31(5), 479-502. doi: 10.1016/j.chiabu.2006.03.012

Finkelhor, D., Hamby, S. L., Ormrod, R. K., \& Turner, H. A. (2005). The JVQ: Reliability, validity, and national norms. Child Abuse \& Neglect, 29(4), 383-412. doi: 10.1016/j.chiabu.2004.11.001 
Gazzaniga, M. S., Ivry, R. B., \& Magnun, G. R. (2002). Cognitive Neuroscience: The biology of the mind. New York: Norton \& Company. doi: $10.1086 / 393043$

Hamby, S. L., Finkelhor, D., Ormrod, R., \& Turner, H. (2004). The Juvenile Victimization questionnaire (JVQ): Administration and Scorig Manual. Durham, NH: Crimes Against Children Research Center. doi: 10.1016/j.chiabu.2004.11.001

Lezak, M. D. (1995). Neuropsychological Assessment. (3rd ed.) New York: Oxford University Press. doi: 10.1590/0101-60830000000049

Lezak, M. D. (2004). Executive functions and motor performance. Neuropsychological Assessment. New York: Oxford University Press.

Medeiros, W., Torro-Alves, N., Malloy-Diniz, L., Minervino, C. M. (2016) Executive functions in children who experience bullyng situations. Frontiers in Psychology, 7. doi: 10.3389/fpsyg.2016.01197

Miyake, A., Friedman, N. P., Emerson, M. J., Witzki, A. H., Howerter, A., \& Wager, T. D. (2000). The unity and diversity of executive functions and their contributions to complex "frontal lobe" tasks: A latent variable analysis. Cognitive Psychology, 41(1), 49-100. doi: 10.1006/cogp.1999.0734

Nuñes Carvalho, J. C. (2016). O impacto dos maus-tratos na cognição e na emoção durante a infância (Tese de Doutorado). Pontifícia Universidade Católica do Rio Grande do Sul, Porto Alegre. Recuperado de http://repositorio.pucrs.br/dspace/bitstream/10923/7974/1/000477905Texto\%2bParcial-.pdf

Oliveira, V., \& Ferreira, D. (2013). Violência e desempenho dos alunos nas escolas brasileiras: Uma análise a partir do SEAB 2011. Revista Econômica, 15(1), 84-114. doi: 10.1590/0103-6351/2591

Organização Mundial de Saúde (2014). Relatório Mundial sobre a Prevenção da Violência. Genova: WHO, 2014.

Sedó, M. (2007). Test de los cinco dígitos. Madrid: TEA Editiones.

Sedó, M., Paula, J.J., Malloy-Diniz, L.F (2015). O teste dos cinco dígitos/FDT. Hogrefe. São Paulo-SP.

Waiselfisz, J. J. (2016). Mapa da Violência 2016. Homicídios por arma de fogo no Brasil. Rio de Janeiro: FLACSO-BRASIL.

Yang, L., Dong, F., Cao, F., Cui,N., Li, J., \& Long, Z. (2013). Poly-victimization and executive functions in junior college students. Scandinavian Journal of Psychology, 54(6), 485-492. doi: 10.1111/sjop.12083

\section{Sobre os autores}

Leandro Jorge Duclos da Costa é professor de Educação Física (UCB/RJ), Pós-Doutor em Psicologia pela PUC/GO e Doutor em Ciências da Educação pela Universidade de Coimbra (Portugal). Atualmente, é docente da Universidade Estadual de Goiás e da Estácio Goiás.

Daniela Sacramento Zanini é Psicóloga (PUC/GO), Pós-Doutora em Psicologia e Doutora em Psicologia Clínica e da Saúde pela Universidad de Barcelona (Espanha). Atualmente, é professora da PUC/GO na graduação e Pós graduação em Psicologia (mestrado e doutorado).

Larissa de Oliveira e Ferreira é Psicóloga (PUC/GO), Mestre em Psicologia pela PUC/GO e especialista em Neuropsicologia. Atualmente, é docente do curso de Psicologia na Faculdade Estácio de Sá Goiás (FESGO).

Karina Junqueira Leão é Psicóloga (PUC/GO), Mestre em Psicologia pela PUC/GO e especialista em Neuropsicologia Clínica pelo IBNeuro. Atualmente é Neuropsicóloga do CRER/GO e Supervisora de Estágio em Neuropsicologia. 